\title{
Anomalies du développement génitosexuel
}

\section{Disorders of Sex Development}

\author{
P.-Y. Mure • C. Grapin-Dagorno \\ (C) Lavoisier SAS 2015
}

La prise en charge des enfants porteurs de troubles de la différenciation génitosexuelle ( $D S D$ : disorders of sex development) [1] a considérablement évolué au cours de ces dernières années. Cette évolution n'est pas uniquement médicale mais elle est également sociétale avec un intérêt grandissant de la société civile pour les questions d'identité sexuelle et de genre.

Nous devons reconnaître au groupe d'experts de la conférence de Chicago de 2005 [1] le mérite d'avoir su proposer une nouvelle nomenclature mieux adaptée à la description de ces anomalies. Cette classification permet une approche précise des diagnostics et apporte une nouvelle terminologie qui supprime certains termes potentiellement stigmatisants comme « ambiguité sexuelle », « intersex » ou « hermaphrodisme ».

Il appartient aux équipes qui travaillent dans le domaine de ces anomalies de proposer une prise en charge optimale de ces enfants et de leurs parents. Compte tenu de la complexité et de la diversité des $D S D$, cela ne peut se faire que par une approche multidisciplinaire hautement spécialisée, et cela dès la détection de l'anomalie. Les progrès du dépistage anténatal conduisent de plus en plus souvent ces équipes à intervenir en période prénatale. Dans les autres situations, elles doivent être impliquées dès la constatation de l'anomalie.

Dans ce dossier thématique, nous décrivons les principes actuels qui vont guider la prise en charge des $D S D$. Nous abordons la description des connaissances modernes du développement génital normal et pathologique. Une mise au point est faite sur la place du diagnostic anténatal et sur les possibilités de prise en charge prénatale dans le cadre particulier des hyperplasies congénitales des surrénales. Il nous a semblé également important de décrire les principes des interventions qui peuvent être proposées, en rappelant pour chacune les indications les plus fréquentes. Enfin, un chapitre est consacré aux problèmes éthiques soulevés par ces anomalies.

Nous ne pouvons qu'insister, encore une fois, sur l'importance de confier ces enfants et leurs parents à des équipes multidisciplinaires expérimentées. La prise en charge de ces anomalies, dont la chirurgie ne représente qu'une facette, doit faire l'objet d'une réflexion adaptée à chaque situation. Les nombreuses questions qui restent posées imposent la création de réseaux spécialisés nationaux et internationaux. Cela permettra de proposer des prises en charge optimales et facilitera les recherches dans ce domaine.

\section{Référence}

1. Hughes A, Houk C, Ahmed SF, et al (2006) Consensus statement on management of intersex disorders. J Pediatr Urol 2:148-62

P.-Y. Mure $(\square)$

Professeur des universités, praticien hospitalier, université Claude Bernard Lyon 1, hospices civils de Lyon, groupe hospitalier Est, hôpital femme mère enfant, service de chirurgie pédiatrique, 59, boulevard Pinel, F-69677 Bron cedex

e-mail : pierre-yves.mure@chu-lyon.fr

C. Grapin-Dagorno $(\square)$

Professeur des universités, praticien hospitalier, université Paris 13, service de chirurgie viscérale et urologique, hôpital Robert Debré, F-75019 Paris

e-mail : christine.grapin@aphp.fr 